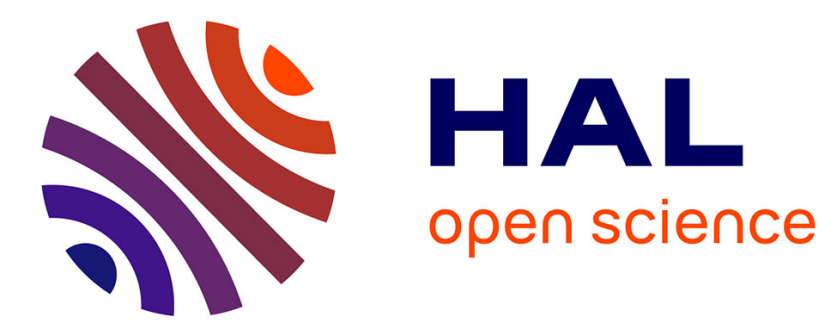

\title{
Les décors peints des petits thermes nord à Saint-Romain-en-Gal (Rhône)
}

\author{
Odile Leblanc, Anne Le Bot-Helly
}

\section{To cite this version:}

Odile Leblanc, Anne Le Bot-Helly. Les décors peints des petits thermes nord à Saint-Romain-en-Gal (Rhône). Gallia - Archéologie de la France antique, 1997, 54, pp.261-275. 10.3406/galia.1997.2999 . hal-01901588

\section{HAL Id: hal-01901588 \\ https://hal.science/hal-01901588}

Submitted on 16 Jan 2020

HAL is a multi-disciplinary open access archive for the deposit and dissemination of scientific research documents, whether they are published or not. The documents may come from teaching and research institutions in France or abroad, or from public or private research centers.
L'archive ouverte pluridisciplinaire HAL, est destinée au dépôt et à la diffusion de documents scientifiques de niveau recherche, publiés ou non, émanant des établissements d'enseignement et de recherche français ou étrangers, des laboratoires publics ou privés.

\section{(이) $\$$}

Distributed under a Creative Commons Attribution - NonCommercial - NoDerivatives| 4.0 


\title{
LES DÉCORS PEINTS DES PETITS THERMES NORD À SAINT-ROMAIN-EN-GAL (RHÔNE)
}

\author{
Odile Leblanc* et Anne Lebot-Helis.**
}

Mots-clés. Rhône, Saint-Romain-en-Gial, thermes, peinture, Diony.sos, décor à réseau, instrumént de musique.

Key-words. Rhône, Saint-Romain-en-Gal, baths, painting, Dionysos, netuork composition, musical instrument.

Résumé. L'étude des enduits peints découtverts dans la démolition des thermes a vévélé sept décors. Trois d'entre enx relèvent du premier état des thermes daté de la fin du Ir s. et les quatre autres sont associés aux pièces chaudes du second état des thermes rénovés au début du III s. I.a majorité des décors présente une organisation en réseau. L'iconographie est en rapport soit avec Dionysos (masque lunaire, syrinx, tympanon, phallus at thyrse), soit avec le monde de l'eau (monstre marin, imitation de coquillages).

Abstract. The study of the mural paintings found in the demolition of the baths has revealed seren paintings. Three of them date from the end of the $2^{\text {mit }}$ century A.D. which corresponds to the first stage of the baths. The other four are associated to the hot rooms of the second stage of the baths restored in the beginning of the $3^{\text {rt }}$ century A.D. The most paintings show a netuork composition. The iconography is related either with Diomysos (face of the moon, syrinx, dulcimer, phallus and thyrse), or with the sea world.

Dans le cadre de la première Loi programme (19881992) sur le Patrimoine national, la mise en valeur du site archéologique de Saint-Romain-en-Gal a donné l'occasion d'achever l'étude des restiges mis au jour une vingtaine d'années auparavant. I.es fouilles programmées de 1988 à 1990 ont notamment porté sur la zone VI, à l'extrémité ouest de l'îlot D situć au nord du site (Savay-Guerraz et al., 1992) (fig. 1). Ces vestiges comprennent des thermes, à l'ouest, et des boutiques à l'est ; ces bâtiments sont séparés par un passage qui relie les deux ruelles parallèles limitant l'îlot au nord et au sud. l'analyse stratigraphique, grâce à plusieurs sondages et à l'étude du mobilier céramique et des monnaies, a permis de reconstituer l'évolution de cet îlot. Créé au début du I" s. après J.-C., il s'est développé au milieu du $\mathrm{I}^{\mathrm{n}} \mathrm{s}$. après J.-C. À cette époque, la partie orientale est occupée par un bâtiment commercial alors que l'espace situé à l'ouest demeurait vide de constructions. Dans le dernier quart du II's. sont installés des petits thermes publics dans la parcelle triangulaire qui est nettement individualisée à l'ouest des boutiques. I.es thermes et les boutiques subissent des réfections au début du III s., avant d'être abandonnés vers le milieu du III's. après J.-C.

\footnotetext{
* Attaché de conservation du Patrimoine, site archéologique de Saint-Romain-en-Gal, BP 3, F-6956(0) Saint-Romain-en-Gal.

** Ingénieur au Service régional de l’Archéologie de Rhône-Alpes, Le Grenier d'Abondance, 6 quai Saint-Vincent, F-69001 lyon.
} 


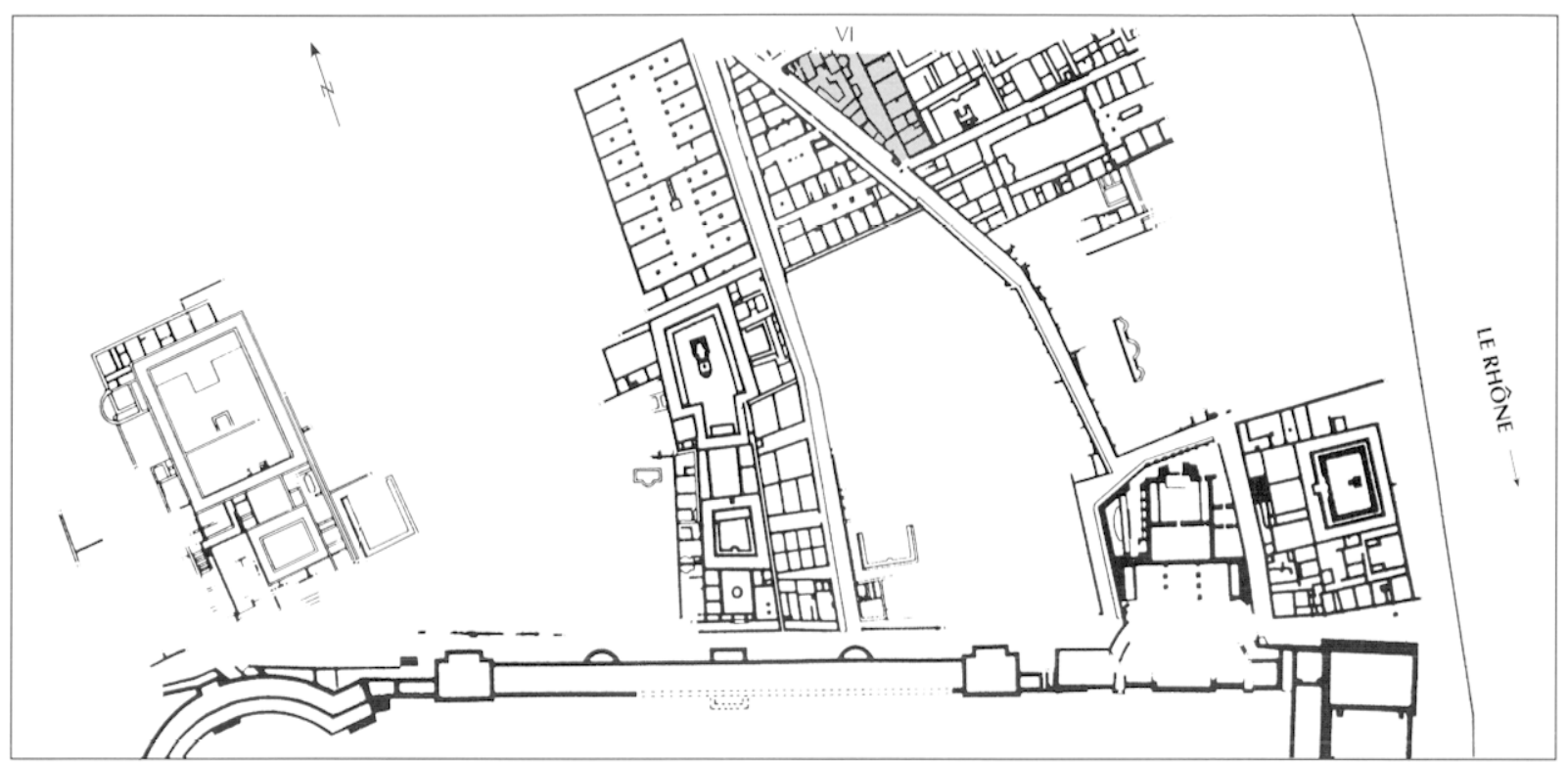

Fig. 1. Plan général du site de. Saint-Romain-en-Gal : zone VI, thermes et bontiques. Échelle : 1/300or (dessin équipe archéologique).

\section{LES THERMES ${ }^{1}$}

L'ensemble s'articule autour d'une petite cour qui desservait au nord les pièces thermales, surélevées, d'une superficie de $140 \mathrm{~m}^{2}$, et de pièces situées au sud.

\section{LE PREMIER ÉTAT DES THERMES}

La cour (n"20), de forme irrégulière, comportait une aire centrale découverte et une galerie sur les quatre côtés (fig. 2). Aux angles, subsistent les traces de dés supportant la toiture.

L'accès se faisait par deux portes : l'une à l'ouest ouvrait sur la voie; l'autre, à l'opposé, munie d'un porche, communiquait avec le passage public. Les deux pièces au sud sont de plain-pied avec la cour. La pointe du bâtiment appartenait peut-ĉtre, dès cette époque, à cet établissement, mais nous ignorons la fonction de ces pièces.

La partie balnéaire comportait un frigidarium ( $\left.\mathrm{n}^{\prime \prime} 30\right)$, qui servait également d'entrée, auquel on accédait

1. La description des vestiges est issue du texte de II. Savay-Guerraz. dans le rapport de synthèse du programme triannuel 1988-1990, SaintRomain-en-Gal (Rhône), fouilles programmées de la Plaine ( $\left.\mathrm{H} 1 \mathrm{n}^{\circ} 82\right)$.

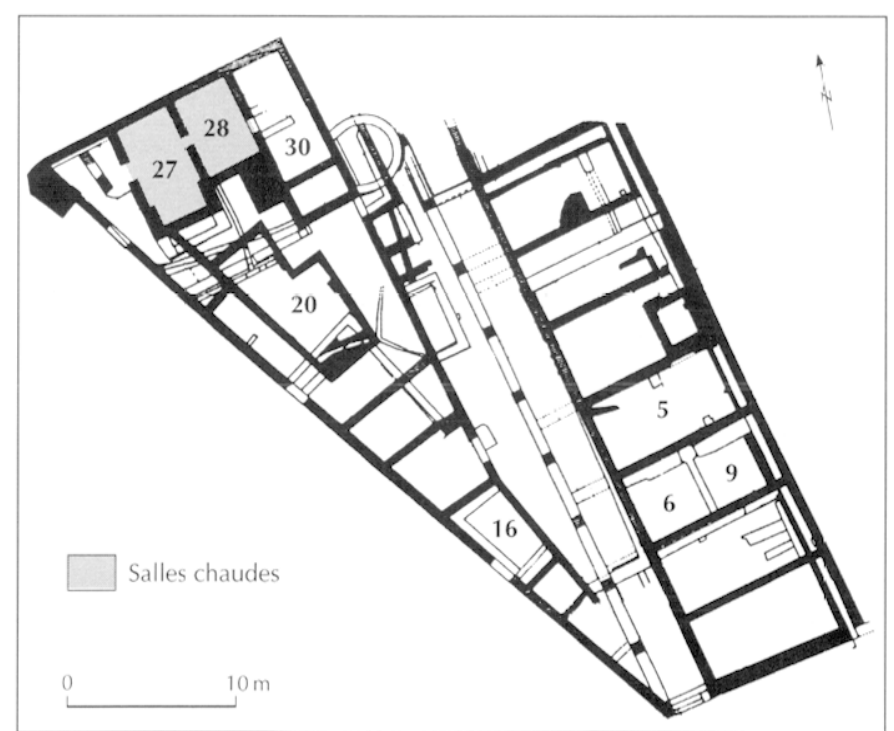

Fig. 2. Plan des vestiges correspondant au premier état des thermes (dessin A.-M. (lappier).

depuis la cour par un escalier. Un bassin peu profond occupait le côté sud. La limite nord de cette pièce est méconnue, l'aménagement ultérieur d'une pièce sur hypocauste ayant fait disparaître la première construction. Les deux pièces sur hypocauste (n" 27 et 28) étaient chauffées à partir d'un foyer placé à l'ouest. L'espace compris entre la façade sur la voie et le mur du caldarium (n"27) forme une sorte de couloir de service accessible 
depuis la ruc. Il ne subsiste qu'une partie du sol de la chambre de chauffe dallée de blocs de molasse. I a réfection des pièces chaudes a fait disparaître leur aménagement d'origine. À l'est, des latrines font saillie sur la laçade.

\section{LE SECOND ÉTAT DES THERMES}

Les reconstructions du début du III's. affectent les thermes et les boutiques (fig. 3). L.es sols des boutiques sont rehaussés à l'aide de remblais provenant de la démolition des pièces chaudes des thermes (fragments de suspensura, tubulures et enduits peints). I.e portique qui bordait en façade les boutiques est supprimé et des pièces en relation avec l'édifice thermal sont installée's dans l'espace ainsi dégagé.

Les pièces thermales sont transformées mais le reste du bâtiment varie peu. Autour de la cour, le sol des galeries a été entièrement refait en béton (terrazzo à inclusions de calcaire). Le sol de la pièce au sud ( $\left.n^{\circ} 16\right)$ a été exhaussé pour y installer des latrines. Ia pièce d'entrée est toujours le frigidarium (n"30) mais la largeur de l'escalier d'accès est réduit. Son sol était à l'origine recouvert d'une mosaïque dont il ne reste que l'empreinte des tessclles dans le béton de support. I.e bassin du premier état est condamné au profit d'une piscine circulaire accolée à la façade (n"31). Dans l'angle nord-est, est créée

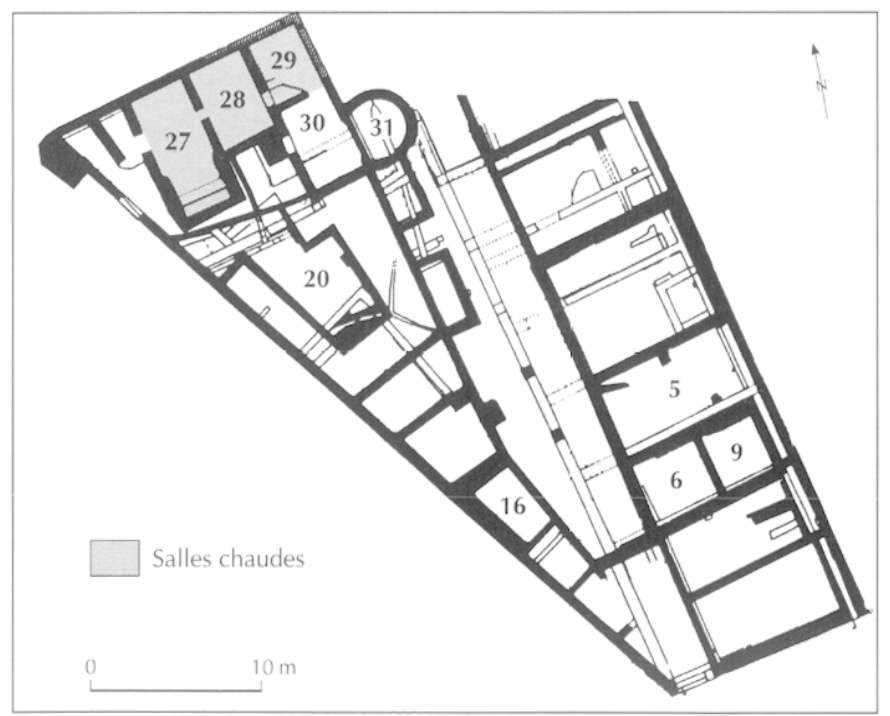

Fig. 3. Plan des vestiges correspondant au second état des thermes (dessin A.-M. Clappier). une petite pièce (n“29) que nous supposons chauffée de manière indépendante car il n'y a aucune communication avec le tepidarium situé à l'ouest ; le foyer se trouverait au nord sous la berme. L'agrandissement du caldarium ( $\mathrm{n}^{\prime \prime 27)}$ de $1,25 \mathrm{~m}$ vers le sud a entraîné une réduction de la cour. Contre la paroi nord de cette pièce est installée, sur la suspensura, une baignoire rectangulaire peu profonde qui présente les traces de placages latéraux et du passage d'une canalisation en plomb. Du côté sud, un rang de briques sur la suspensura délimite un espace carré aménagé à la faveur de l'agrandissement du caldarium. Dans le tepidarium (n*28), la suspensura, découverte entièrement effondrée, ne révèle aucune trace d'aménagement particulier. I es couches d'abandon dans ces pièces chaudes renfermaient de nombreux fragments d'enduits peints mêlés à la démolition.

\section{LES DÉCORS PEINTS DU PREMIER ÉTAT DES THERMES}

Les fragments de peintures étudiées proviennent de remblais apportés dans les boutiques (pièces 5,6 et 9 ) lors de la reconstruction du dernier état. Ils correspondent à trois décors à réseaux sur fond blanc. Le premier (n'VI-5) représente une surface de $4 \mathrm{~m}^{2}$ environ; il est très fragmentaire mais assez bien conservé. Le deuxième (n"VI-6) est un badigeon de chaux sur l'enduit du premier décor qui a été repeint. Ce badigeon n'était pas conservé sur l'ensemble des fragments du décor 5 et les motifs sont évanescents. Ie troisième ( $n^{\circ}$ VI-7) a un support en mortier de tuileau, et il est conservé de façon très fragmentaire sur une surface de $0,8 \mathrm{~m}^{2}$ environ.

\section{DÉCOR VI-5}

La trame de ce décor couvrant est composée de carrés, d'environ 3 pieds de côté $(90 \mathrm{~cm})$, disposés en quinconce (fig. 4). Des motifs végétaux en croix, peints sur les médianes, les divisent en quatre carrés décorés d'un cercle qui délimite le décor figuré.

I es côtés des carrés de base sont dessinés par une tige rectiligne ornée de petites feuilles alternées vertes. Les angles sont soulignés par des fleurons rouges à quatre pétales allongés ou cordiformes. La disposition en 


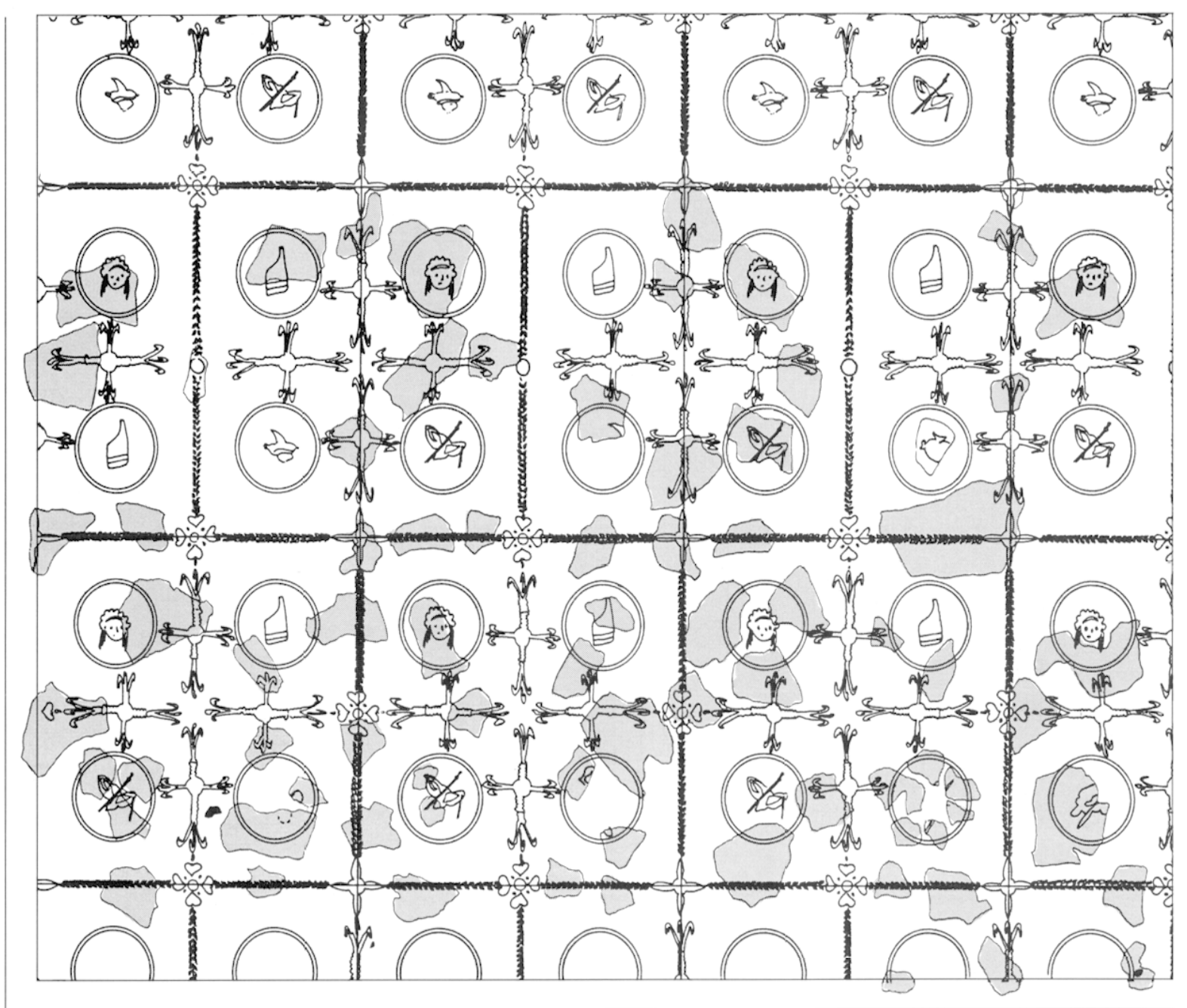

Fig. 4. Restilution de la trame du décor VI-5, les fragments conservés sont tramés (dessin O. Leblanc).

quinconce des carrés de base est soulignée par une alternance des types de fleurons.

Les rameaux sont disposés en croix, leur centre est marqué par un rond vert rehaussé de rouge. Le grand bras de la croix, qui représente trois feuilles issues d'une tige ondulée, est en relation avec l'un ou l'autre des fleurons. Le petit bras, formé de trois feuilles vertes, vient buter contre les cercles de $20 \mathrm{~cm}$ de diamètre qui sont dessinés par un filet rouge de $1 \mathrm{~cm}$ de large. Leur centre est orné d'un objet stylisé. Quatre motifs différents ayant été retrouvés en plusieurs exemplaires, il est possible qu'ils aient décoré chacun des cercles du carré de base.

Ces motifs sont traités de façon sommaire, comme des ombres, dans les tons noir, gris et rouge. Une tête ronde, dont le modelé de la face est rendu par un dégradé de gris rehaussé de touches blanches, est présente en six exemplaires. Un bandeau blanc souligne la racine des cheveux et de chaque côté du visage pendent deux rubans gris qui suggèrent que l'objet est accroché (pl. I, n"1). Ces figures sont généralement définies comme des masques lunaires (Barbet, 1983a). L'objet de forme grossièrement trapézoïdale dans des tons gris foncés parfois rchaussés de rouge (pl. I, n"2) est interprété comme une flûte de Pan (4 exemplaires) ( $c f$. infra, p. 273, addendum de A. Belis). Les deux derniers motifs ne sont pas directement reconnaissables : l'un (4 exemplaires) représente une tige ou un bâton enrubanné de couleur sombre devant un aplat en forme de croissant de couleur rouge ombré de noir (pl. I, n"3) ; l'autre pcut être lu comme une sorte de baguette à l'extrémité renflée disposéc 
devant un ovale noir terminé par une boucle. Cet objet était certainement destiné à être suspendu. L'un des deux exemples conservés est incomplet (pl. I, n*5) et l'autre est très effacé par le traitement de surface qu'a subi la peinture pour recevoir un nouveau décor ( $\mathrm{pl}$. I, n"4).

Le thème qui sert de clef de compréhension à cet ensemble iconographique est d'inspiration dionysiaque, car la flûte de Pan, souvent associée dans la peinture murale à des masques lunaires, est un des symboles de ce culte. Dans ce registre, nous proposons d'interpréter les deux derniers motifs respectivement comme : un thyrse cnrubanné posé devant un phallus² et un gong accompagné d'un instrument de percussion ".

Les tracés préparatoires ont été réalisés à l'aide d'une cordelette dans le mortier frais. Ils sont visibles sur les fleurons à longs pétales où ils se croisent, sur certaines lignes de feuilles alternées, sur l'axe des fleurons cordiformes en relation avec la tige de feuilles alternées et sur l'axe long de cortains rameaux de la croix. I.e schéma préparatoire est donc un quadrillage de $90 \mathrm{~cm}$ de côté dont les angles sont soulignés par des fleurons à longs pétales et les côtés par des tiges de feuilles. I a présence de tracés sous les rameaux et sous un axe des fleurons cordiformes, ainsi que l'absence de ces tracés sous des tiges de feuilles, démontrent que la construction de la composition géométrique n'a pas été faite à partir d'un simple quadrillage.

Ce décor à réseaux est encadré par une bande rouge bordeaux située dans les angles du mur ; l'arrachement, à angle droit, du mortier visible sur la couche picturale indique un retour de la peinture. I.es quelques fragments, qui présentent une bande dégradée de gris légèrement courbe en contact avec les tiges de feuilles alternées, indiqueraient l'existence d'une lunette. Les fragments chanfreinés, qui sont décorés d'une bande rouge bordeaux limitant un champ blanc, témoignent d'une ouverture dans la paroi.

Le mortier est composé de trois couches sous la pellicule picturale : ocre jaune pâle de 0,8 à $1 \mathrm{~cm}$ avec du sable fin de rivière et du quartz, très compact, beige de 2 à $2,5 \mathrm{~cm}$ avec du sable plus grossier et friable, enfin une

2. Nous remercions $M$. Fuchs qui nous a proposé cette interprétation. 3. Nous proposons un gong plutôt qu'un tympanon sur les conseils de A. Belis, qui voit dans cette figure un objet plein plutôt qu'une peau tendue sur une armature couche rose $(1,5 \mathrm{~cm})$, en mortier de tuileau, qui présente des empreintes de tubulures.

I a couche picturale est peinte à fresque, elle porte quelques traces d'effacement, notamment sur les motifs ornant les cercles (pl. I, n"4). Cé décor a été recouvert entièrement d'un badigeon de chaux sur lequel a été repeint un nouveau décor.

\section{DÉCOR SUR BADIGEON : DÉCOR VI-6}

Ce nouveau décor reprend les même types de motifs mais ils sont traités de manière plus gracile (fig. 5) : un cercle de 28 à $30 \mathrm{~cm}$ de rayon dessiné par un fin filet ocre-rouge $(0,2 \mathrm{~cm}$ de large), des tiges portant des bouquets de feuilles vert-marron et de fleurs ocre-rouge, des fleurons cordiformes ocre-rouge el une bande d'encadrement rouge bordeaux. Il nous est impossible de pro-
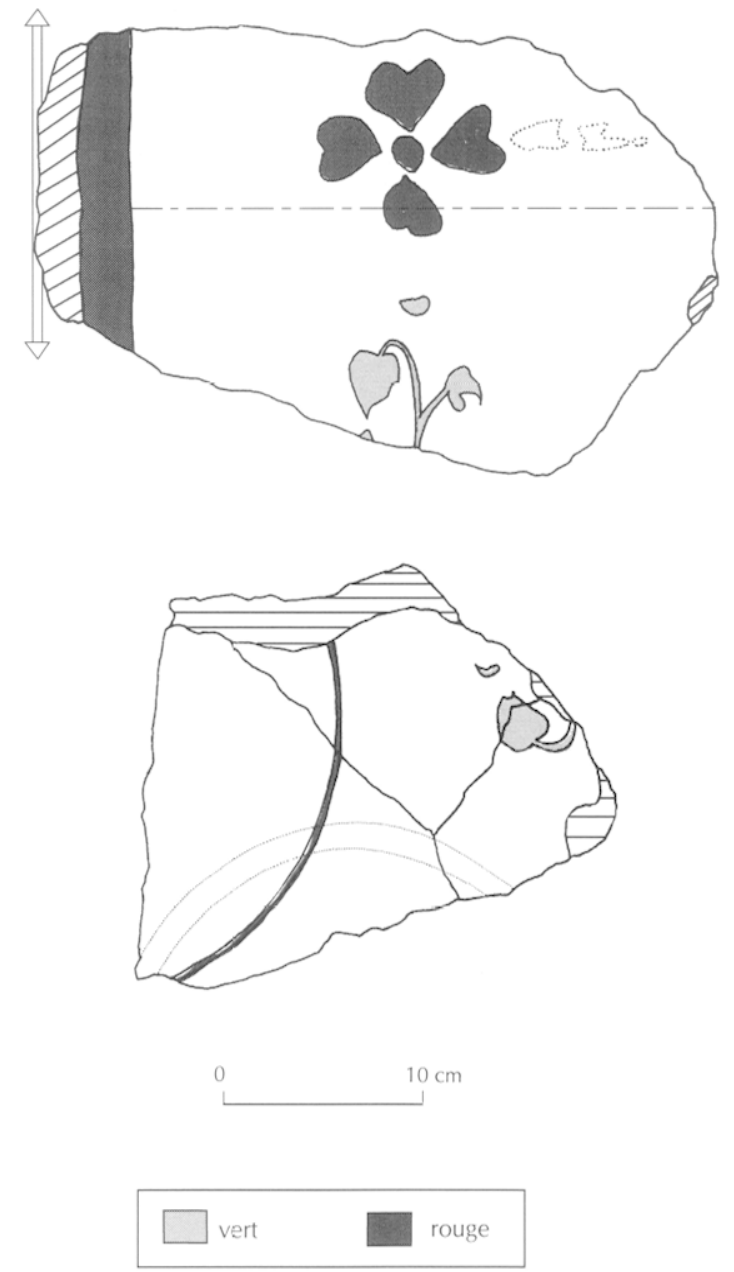

Fig. 5. Décor VI-6, tramé ; décor VI-5, pointillé; tracés préparatoires, tiret-point (dessins O. Leblanc). 
poser une restitution du décor, mais son organisation est différente et complètement indépendante du décor précédent dont on discerne encore les motifs et le tracé préparatoire sous le badigeon. Il ne s'agit donc pas d'une simple réfection mais d'une nouvelle phase décorative.

\section{DÉCOR SUR MORTIER DE TUILEAU : DÉCOR VI-7}

Deux ensembles de fragments-clés nous permettent de proposer un schéma pour la trame. Des feuilles allongées de couleur ocre-rouge sont disposées en croix ; sur la bissectrice des angles droits ainsi formés sont peintes des tiges de feuilles alternées vertes (fig. 6, n” 1 ). L'empreinte d'une cordelette indique un tracé préparatoire, au moins sur un axe des fleurons rouges. Des bouquets composés de pétales trilobés rouges et de feuilles vertes sont disposés sur une tige qui porte des nodules (fig. 6, n" 2). Une de ces tiges décorées forme un angle de $45^{\circ}$ avec une bande rouge (fig. $6, \mathrm{n}^{\circ} 3$ ) qui devait souligner un bord de paroi ; d'autres fragments de bande rouge présentent des arrachements caractéristiques d'angles rentrants. Nous supposons que l'organisation du décor est basée sur un quadrillage ponctué de fleurons rouges et souligné par les tiges de feuilles alternées, les bouquets sur tiges pouvant être disposés sur les diagonales ou les médianes des carrés.

Le mortier de support est constitué d'une couche de tuileau d'une épaisseur variant de 2 à $3 \mathrm{~cm}$ sous la couche picturale faite de chaux blanche pure. La surface du décor présente plusieurs traces de piquetage (fig. 6, n"2).

\section{Conclusion}

La nature des mortiers (tuileau, négatifs de tubulures) suggère que les enduits peints, découverts dans les boutiques situées vers les thermes nord, proviennent de ceux-ci. La contemporanéité et la cohérence du dernier état de l'ensemble boutiques-thermes expliquent aisément ce transport des décombres sur quelques dizaines de mètres.

Le piquetage du décor sur tuileau indique une réfection, comme celle qui a été observée sur le décor VI-5, ce qui tendrait à prouver leur appartenance à la première phase d'une même pièce. Cette hypothèse semble confirmée par la similitude des motifs de ces deux décors. On peut imaginer qu'ils ornaient un même espace divisé en deux parties qui pouvaient avoir des fonctions diffé-

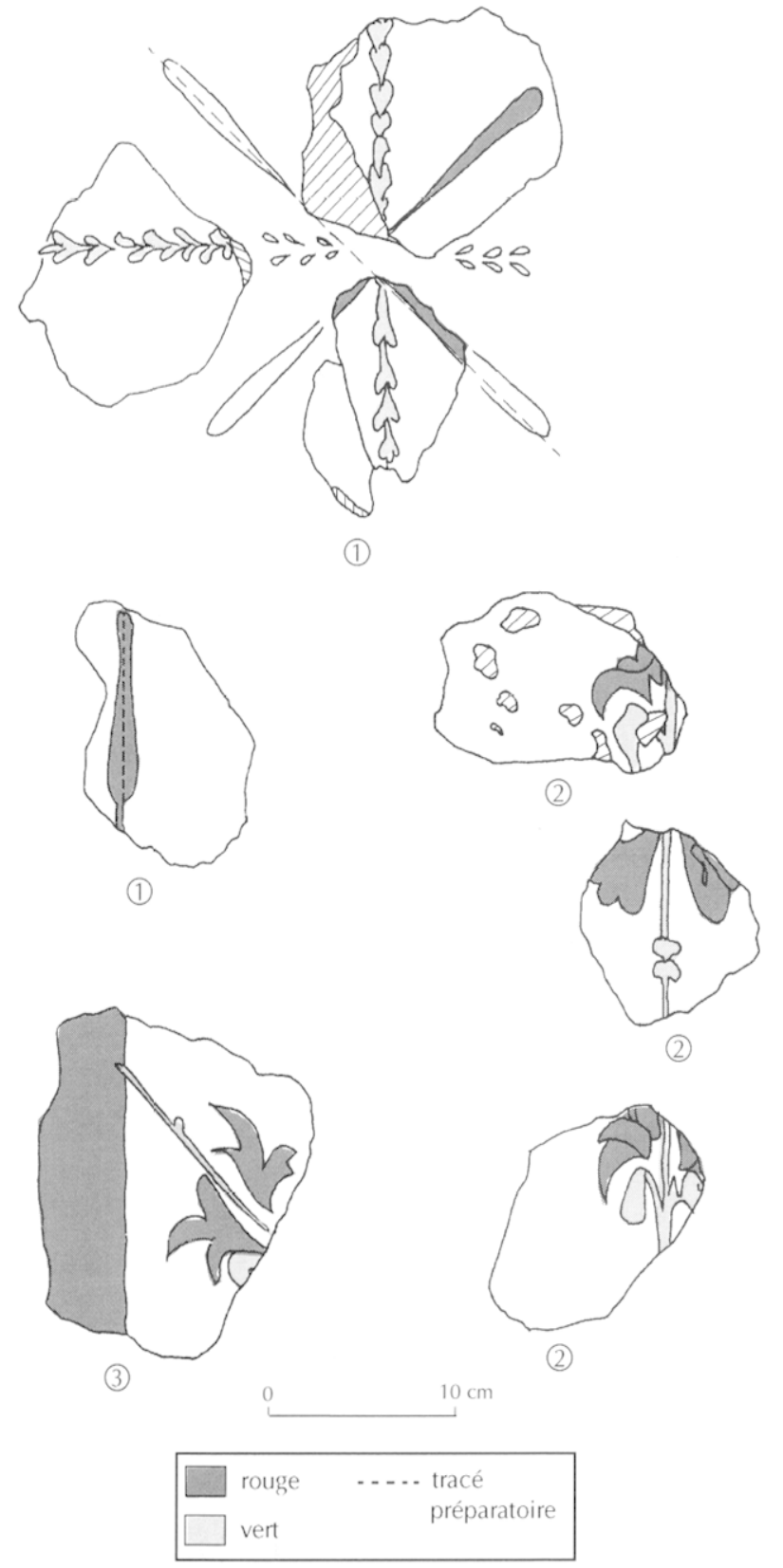

Fig. 6. Décor VI-7: 1, fleuron en croix; 2, lige avec bouquets ; 3, angle de paroi (dessins (). Leblanc).

rentes, par exemple une salle et une piscine. Le maillage très large de la trame de base du décor VI-5 paraît correspondre à une grande pièce, les empreintes de tubuli désignent une salle chauffée, tandis que le support en tuileau du décor n" 7 implique une pièce en contact avec l'eau. Ces décors proviennent très probablement du caldarium (n"27) qui présenterait, dès le premier état des thermes, une exèdre où pourrait avoir été installée une baignoire. 


\section{LES DÉCORS PEINTS DU SECOND ÉTAT DES THERMES}

Les peintures ont été recueillies dans les pièces chaudes des thermes, en 1967, lors du dégagement extensif du site, puis le Groupe archéologique de Vienne a repris, en 1975, la fouille des pièces 27 et 28 jusqu'au niveau du sol et a recueilli les fragments d'enduits peints mêlés à la démolition. Ces peintures, étudiées en 1978 par les archéologues bénévoles du Touring-Club de France, ont fait l'objet d'un rapport ${ }^{4}$. Lors de la campagne de fouilles réalisée en 1990, l'équipe archéologique départementale a amorcé le dégagement de la pièce 29 dans l'angle nord-est des thermes et découvert des fragments de peinture dans les couches de démolition (VI-29-1 et 2). I es cinq ensembles cohérents qui ont été mis en évidence permettent de proposer des restitutions pour le décor des deux pièces chauffées.

\section{DÉCOR AUX OCTOGONES : DÉCOR VI-4}

Les $9 / 10^{*}$ des fragments composant ce décor ont été ramassés entre 1975 et 1976 dans les couches de démolition du caldarium (pièce n* 27) parmi les éléments de suspensura, essentiellement à l'emplacement de l'hypocauste effondré, c'est-à-dire au centre de la pièce (fig. 3). Un décor à réseaux, composé d'octogones adjacents déterminant des carrés sur la pointe, et une imitation de coquillages et de mosaique sont associés dans la décoration de cette pièce.

Les octogones $(18 \mathrm{~cm}$ de côté vers l'intérieur du motif) sont dessinés par une bande verte ou bleue $(4 \mathrm{~cm})$ soulignée à l'extérieur d'un filet noir et d'une imitation de moulure $(6 \mathrm{~cm})$ réalisée par une succession de filets blancs, bruns et jaunes. Leur fond est blanc, un filet rouge les souligne à l'intérieur (fig. 7). Leur centre est orné de scènes : personnages qui pourraient être des Amours, les taches de couleur derrière les épaules représenteraient des ailes (pl. II, n" 1 et 2) ; personnages portant un plateau de fruits (pl. II, n" 3 et 10); lutteurs affrontés : la position des personnages correspond à la représentation classique de préparation à l'affrontement

4. D. Meyrand, B. Vidal, F. Bertholon et M.-J. Bodolec, Étude sur les peintures murales des thermes de Saint-Romain-en-Gal, archives du site. dans la lutte ${ }^{5}$ (pl. II, $\left.\mathrm{n}^{\circ} 4\right)$. Ces figures sont colorées avec une dominante brune rehausséc soit de vert, soit de bleu. Les carrés sont dessinés par une bande ocre-rouge soulignée à l'intérieur d'un filet rouge et à l'extérieur d'un filet ocre-rouge plus foncé $(6 \mathrm{~cm})$ et d'une imitation de moulure réalisée par une succession de filets blancs, bruns et jaunes $(4 \mathrm{~cm})$. Leur centre est blanc, décoré d'un fleuron à huit pétales, alternativement vert clair et foncé. Plusieurs fragments de peintures montrent des octogones coupés par une bande rouge foncé (largeur minimum de $7 \mathrm{~cm}$ ) et des angles de carrés viennent buter contre cette même bande rouge. Le profil en biseau du mortier et l'angle rentrant formé par la couche picturale de plusieurs de ces enduits indiquent un angle de paroi. Un fragment de bande rouge en rapport avec les octogones souligne un angle convexe de la paroi, ce qui implique une ouverture.

Les fragments qui représentent des imitations de coquillages et de mosaiqque sont peu nombreux (Barbet, 1983b). Une bande rouge foncé est décorée d'un motif linéaire composé d'une alternance de coquilles du type Cardium dans les tons blanc-beige et de carrés blancs épars représentant des tesselles de mosaïque (fig. 8). Cette bande est peinte sur un angle convexe de la paroi qui indique une ouverture. Elle est soulignée d'un côté par une ligne continue de tesselles de mosaíque peintes en blanc qui délimite un champ bleuté avec un réseau régulier de traits noirs. D'autres fragments présentent ce même genre de décor dans les tons bleus et verts (pl. II, n*5-9). Il s'agit probablement d'un opus musivum en trompe-l'œil dont le sujet n'a pu être lu, mais qui doit être en rapport avec des scènes marines (Barbet, 1983b).

\section{LES TRACÉS PRÉPARATOIRES}

Les empreintcs d'unc cordclcttc, espacécs d'unc dizaine de centimètres, sont visibles le long des filets marron qui marquent les côtés adjacents des octogones et sur une diagonale du carré sur la pointe. Elles permettent de proposer la base de l'organisation du tracé préparatoire : les doubles lignes qui indiquent la largeur de la moulure sont tracées sur des parallèles espacées de 2 pieds

5. Le fragment où était représentait ce dernier motif a malheureusement été égaré. 


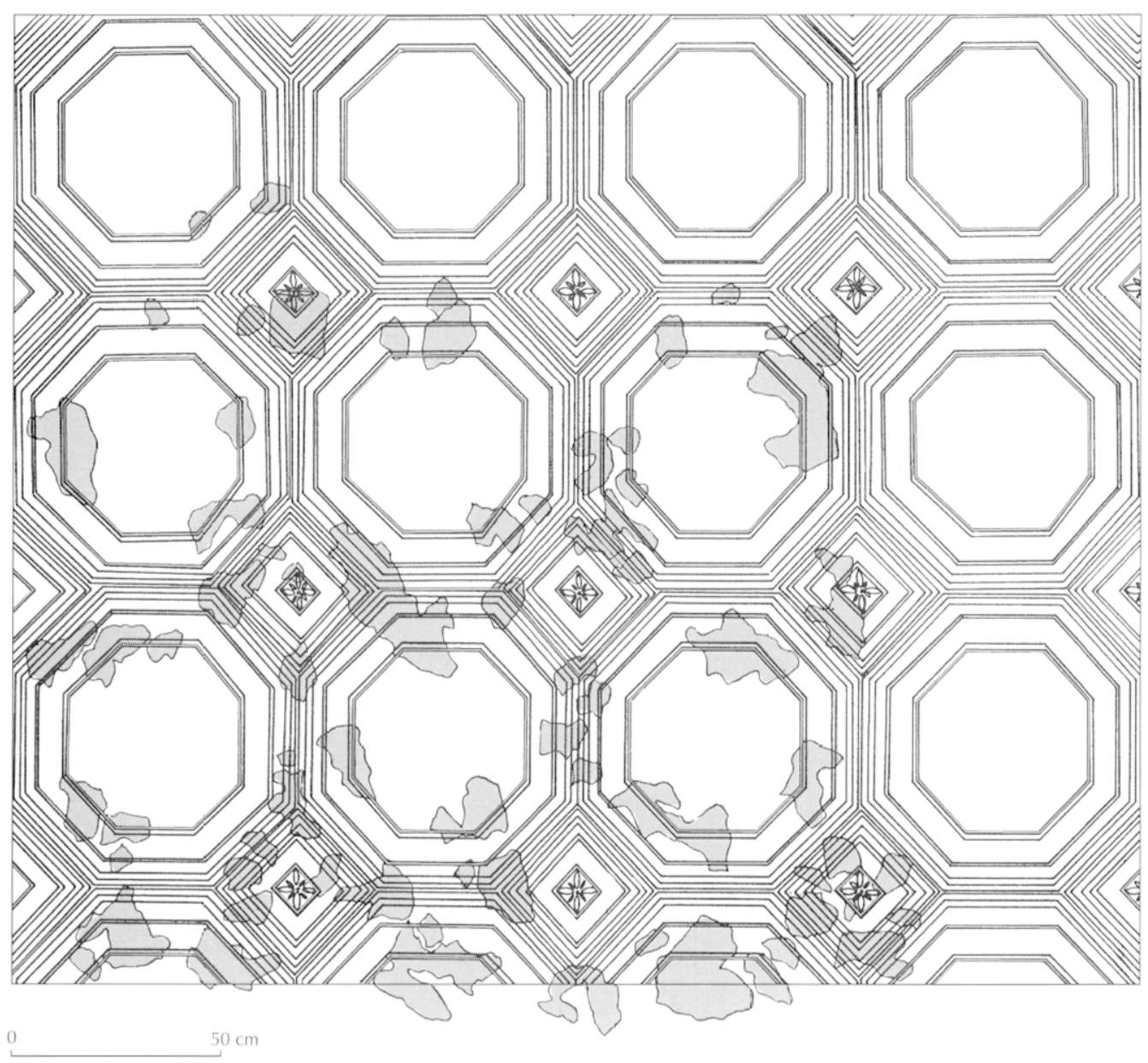

Fig. 7. Restitulion de la trame du décor aux octogones, les fragments conservés sont tramés (dessin (). L.eblanc).
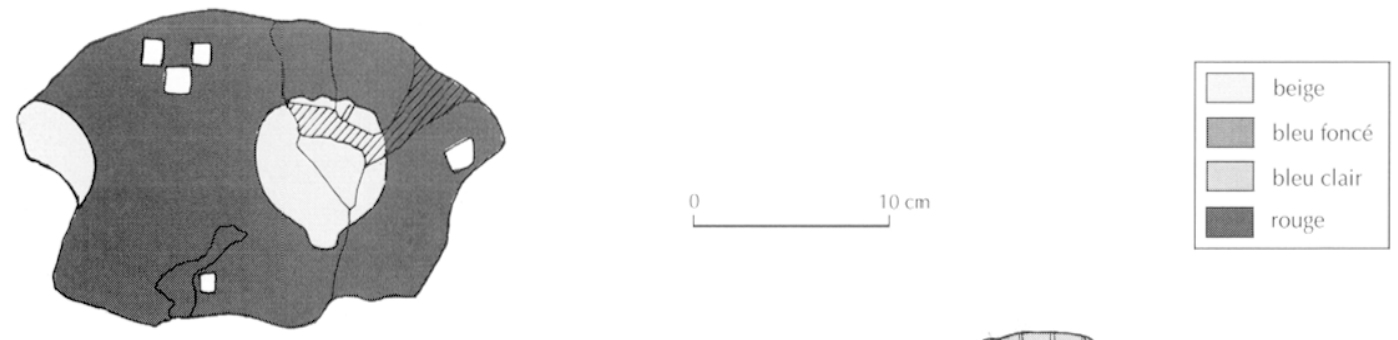

(1)
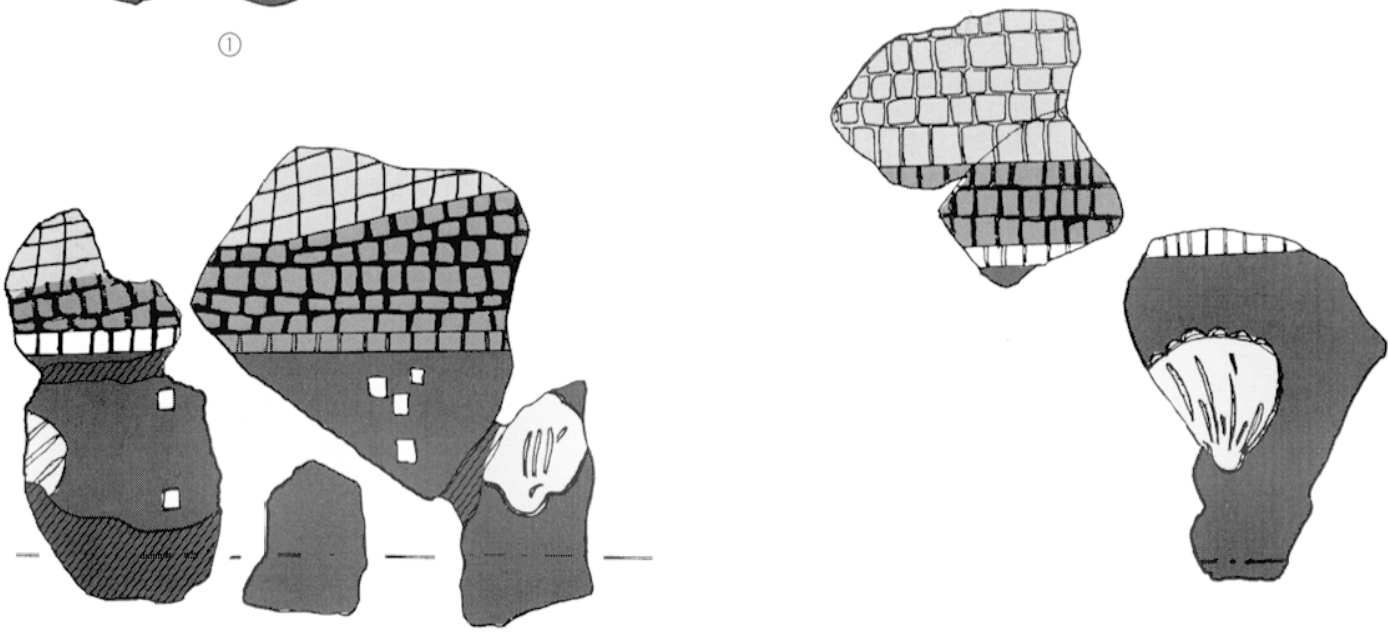

(2)

(2)

Fig. 8. Décor VI-4 : 1, bande d'encadrement avec négatif de coquillages incrustés ; 2, bande d'encadrement avec coquillages peints et imitation de mosä̈que; arête de l'angle, tiret-point (dessins M.J. Bodolec). 

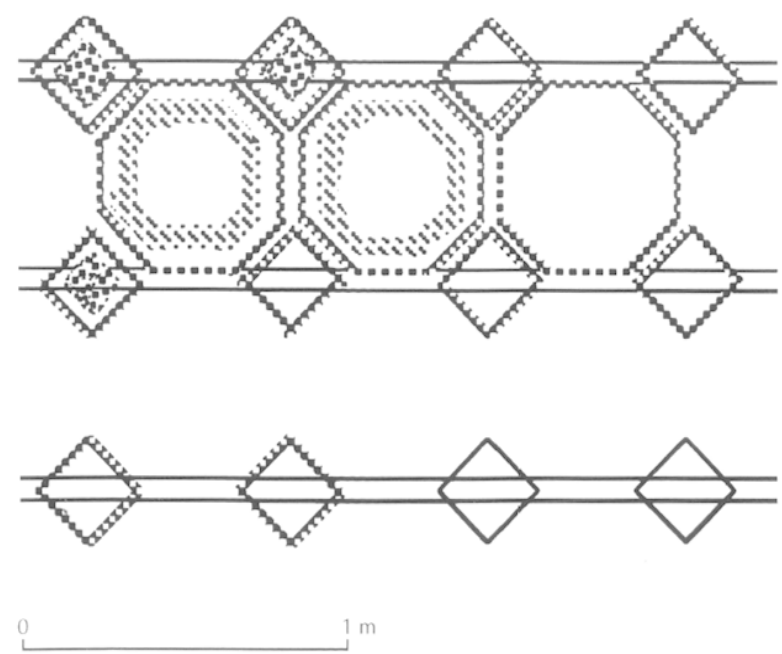

Fig. 9. Schéma de réalisation du décor aux octogones a partir de la trame (dessin O. Leblanc).

$(60 \mathrm{~cm})$ selon un des axes de la paroi ; cette distance de 2 pieds étant fournie par la largeur des octogones.

Des traces de pointe de compas sont visibles sous le fleuron central des carrés dont le périmètre est marqué d'une ligne incisée sous les filets marron. Ces empreintes permettent de déterminer l'axe perpendiculaire du quadrillage. L'espace entre deux carrés est marqué à l'aide d'un compas et le pourtour est dessiné pour déterminer deux côtés de l'hexagone (fig. 9).

I.e mortier est composé de trois couches, décrites de la couche picturale vers le mur :

- la première, de $0,2 \mathrm{~cm}$ d'épaisseur, est un mélange de chaux et de sable de rivière de granulométrie fine ;

- l'épaisseur de la deuxième varie de 2,3 à $4,3 \mathrm{~cm}$, elle contient du sable de granulométrie plus grossière ;

- la troisième ne subsiste que sous forme de traces de mortier de tuileau.

\section{LA RESTITUTION DU DÉCOR DE LA PIÈCE}

Bien que nous n'ayons pas retrouvé d'empreintes de tubuli, le lieu de découverte et la composition du mortier suggèrent que ces deux ensembles ont appartenu à la décoration du caldarium (fig. 3, n"27). La planéité des fragments et la présence d'angles convexes, qui indiquent une ouverture, permettent de situer cette peinture sur une paroi verticale. Ce genre de décor, qui existe dès le $\mathrm{I}^{\mathrm{er}} \mathrm{s}$. dans la pièce monochrome de la maison aux
Amours Dorés à Pompéi, est assez répandu à la fin du II" et au début du III's. (Drack, 1982) ".

Nous pouvons donc imaginer, sur les murs du caldarium, un décor couvrant, imitant des caissons alternativement verts et bleus (fig. 10). L'effet de relief, que l'on attendrait plutôt sur un plafond, est rendu par un dégradé de couleurs qui imite une moulure de stuc autour des tableaux figurés. Le décor à scène marine imitant la mosaique, encadré d'une bande rouge foncé avec coquillages, ornerait les murs de l'exèdre avec piscine située au sud. Nous n'avons aucun élément de la partie basse : nous supposons soit des placages de marbre, soit un enduit de tuileau peint en rouge $\left(0,50 \mathrm{~m}^{2}\right.$ de ce type d'enduit provient de cette zone).

\section{DÉCOR AUX HEXAGONES : DÉCOR VI-1}

L.es fragments proviennent de la pièce n" 28 (fouilles 1975-1978) ct de la pièce n" 29 couches 1 et 2 (fouilles 1989-1990) (fig. 3). Ce décor est composé d'une trame à base d'hexagones qui est encadrée d'une bande verte soulignée d'une moulure en trompe-l'œil.

Une bande bleue, de 4 à $4,5 \mathrm{~cm}$ de large, dessine des hexagones sur fond blanc, la longueur des côtés des hexagones, à l'intérieur de la moulure, varie entre 15 et $22 \mathrm{~cm}$. Ils sont soulignés à l'intérieur par une série de

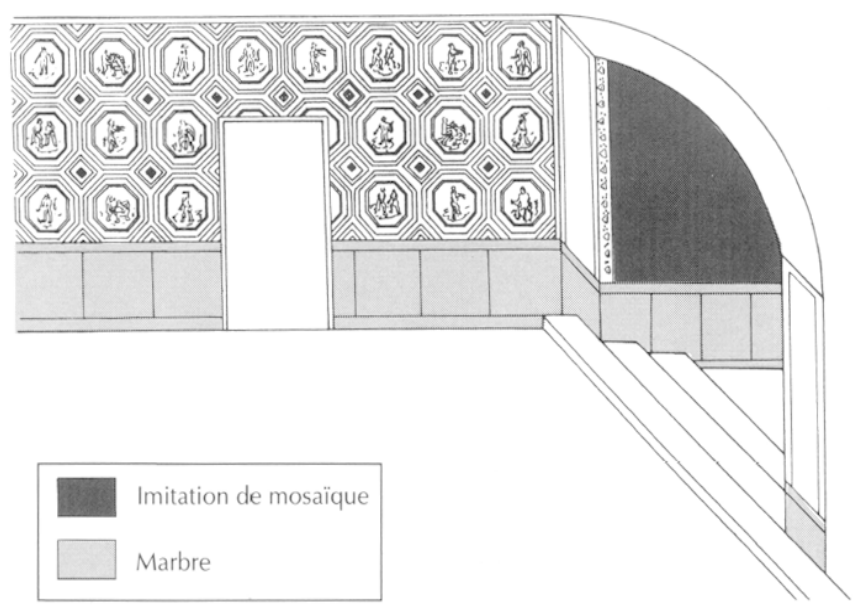

Fig. 10. Proposition de restitution du décor du caldarium au début du III s. après J.-C. (dessin O. Leblanc).

6. De nombreux autres exemples provenant de différentes régions sont présentés dans les Actes des Journées de Paris 1982, sur la peinture murale romaine dans les provinces de l'Empire. 

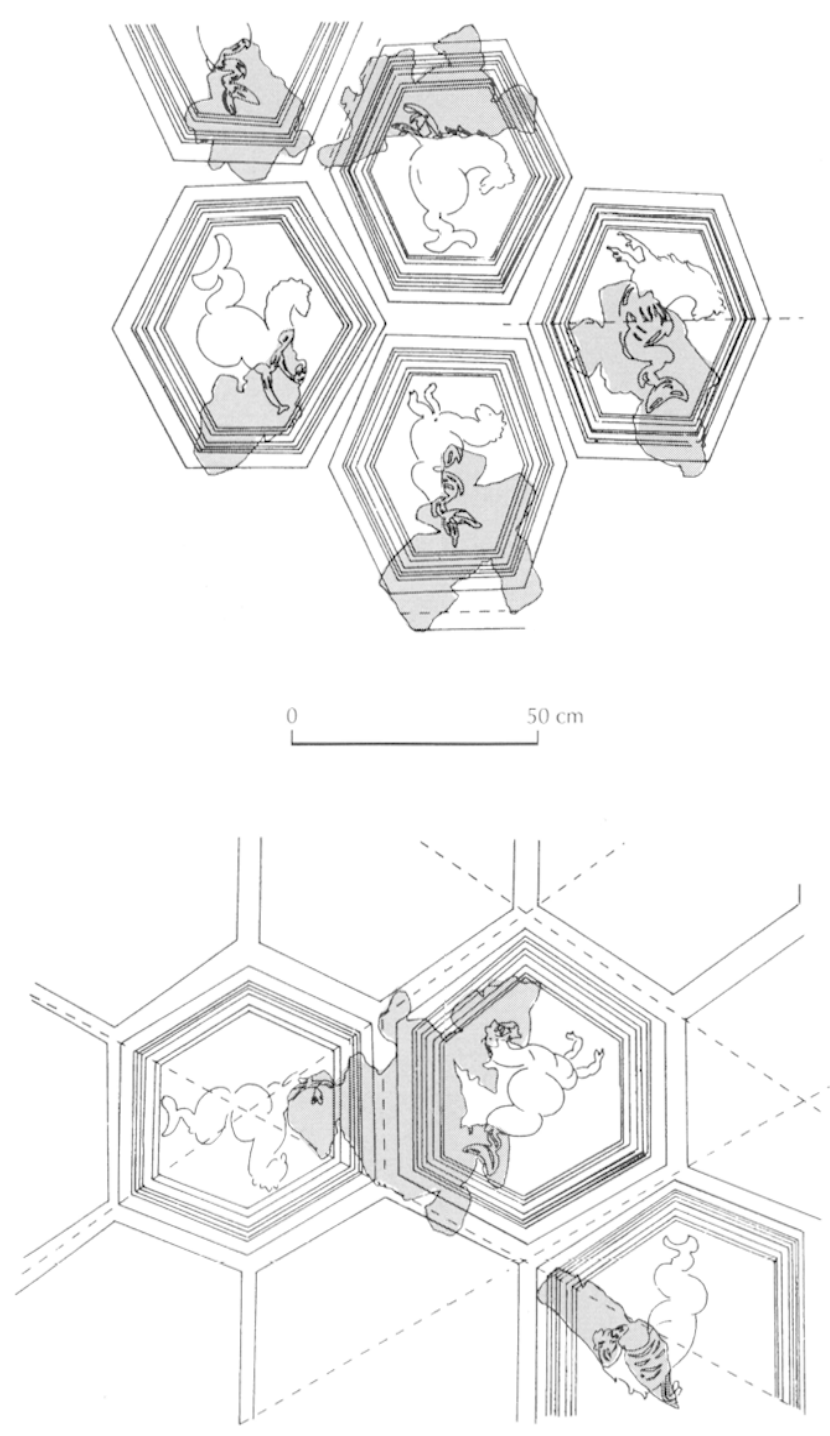

Fig. 11. Restitution de la trame du décor aux hexagones (décor VI-1) : les fragments conservés sont tramés, les tracés préparatoires sont en pointillé (dessins M.J. Bodolec et O. Leblanc).

filets dans les tons ocre jaune clair et foncé qui peuvent être assimilés à une moulure fictive. L eur centre est orné de figures marines dans les tons ocre, jaunes et verts : tête de panthère ou de cheval, pattes antérieures d'animaux, queue de poisson (pl. I, n"6).

La valeur des angles conservés $\left(60^{\circ}\right.$ et $\left.120^{\circ} \pm 10^{\circ}\right)$ indique des hexagones, mais la variation de ces angles et les différentes longueurs des côtés impliquent une distorsion de la figure de base (fig. 11). Cette déformation, liée à la nćcessité d'appliquer ce schéma à un support concave attesté par plusieurs fragments d'enduits peints qui présentent une faible courbure, a été renforcée. Elle accentue ainsi l'effet de perspective qui donne l'illusion d'une plus grande profondeur de la voûte qui supportait ce décor.

Des tracés préparatoires visibles au milieu de certaincs bandes bleues et sur les diagonales de certains hexagones laissent supposer un tracé directeur à base de losanges aplatis. Il est probable que les deux diagonales de la voûte aient été fixées, puis que des parallèles aient été développées à des intervalles allant en diminuant dans le sens de l'axe de la voûte.

Ce motif de caisson est encadré par une bande verte suivie d'un champ blanc. Une bande verte similaire est associée à deux types de moulures fictives. Le premier type est rendu par une succession de bandes et filets dans les tons ocre sur laquelle sont peints des oves schématisés blancs et noirs. Le profil du mortier indique un angle vers le côté éclairé de la moulure (blanc et ocre jaune clair) (fig. 12, n*1) qui correspond à un joint de journée ${ }^{\top}$. Cette moulure se placerait en base de voûte sur le côté longitudinal. L'autre type de moulure est constitué d'une succession de bandes et filets ocre clair et foncé en arc de cercle qui se situerait dans la lunette (fig. 12, n"2).

Le mortier est composé de trois couches sous la pellicule picturale :

- la première, de 0,2 $\mathrm{cm}$ d'épaisseur, est un mélange de chaux et de sable de rivière de granulométrie fine ;

- la deuxième a une épaisseur qui varie de 2,8 à $3,3 \mathrm{~cm}$ avec un sable de granulométrie plus grossière ;

- la troisième de $1,5 \mathrm{~cm}$ est un mortier de tuileau.

Des négatifs de tubuli sont visibles sur le revers d'un des fragments-clés; leur organisation incohérente nous les fait interpréter comme une réparation de la voûte.

\section{DÉCORS DE FAUX MARBRES : DÉCORS VI-2 ET VI-3}

Deux types d'imitations de marbre à fond beige et rehaut ocre rouge ont été reconnus. Ils ornaient certainement des panneaux limités par un double filet noir et une bande verte (fig. 13, n"1). Sur un fragment, la bande verte est suivie d'une succession de filets ocre jaune (fig. 13, n"3) et un fragment présente la relation à angle droit de filets ocre avec une autre moulure (fig. 13, no2).

7. C'est une limite régulière visible dans l'épaisseur du mortier qui indique que l'artisan peintre a arrêté de poser son enduit frais car il avait assez à peindre pour la journée. 


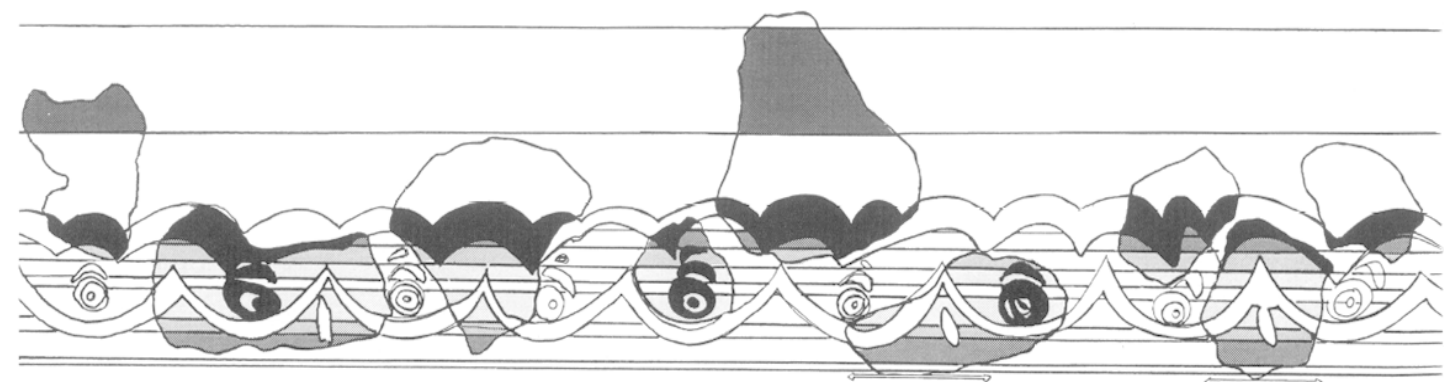

(1)
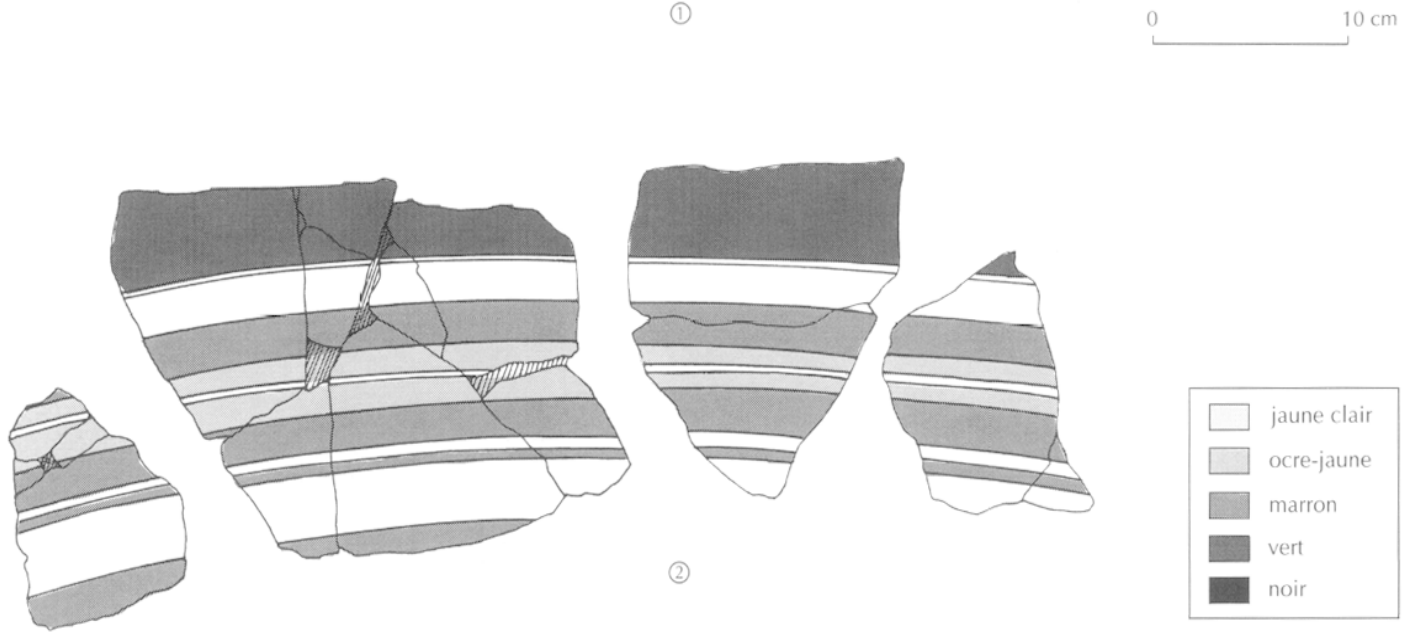

Fig. 12. Décor VI-1 : 1, imitation d'une moulure simple rehaussée d'oves; 2, imitation d'une moulure courbe (dessins M.J. Bodolec el (O. Leblanc).

Ces imitations de marbre représenteraient des orthostates en zone II : la bande verte correspondant aux listels et aux filets ocre d'un pilastre, la moulure fictive serait une corniche d'entablement.

Les mortiers sont similaires à ceux du décor précédent (VI-1).

\section{LA RESTITUTION DU DÉCOR DE LA PIÈCE}

La similitude des mortiers et de la palette des couleurs ainsi que le lieu de découverte permettent de rattacher les ensembles 1,2 et 3 à la décoration du tepidarium (pièce $\mathrm{n}^{\circ} 28$ ). Elle serait composée de grands orthostates en faux marbre séparés par des pilastres dans la zone médiane des parois et d'un décor à caissons hexagonaux avec des figures de monstres marins sur la voûte (fig. 14).

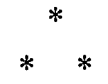

L.es décors présentés s'inscrivent dans une mode picturale de décors à réseaux de la fin du II et du début du III" s. Les décors à caissons sont la transcription picturale de système décoratif en stuc connu dans le monde romain et particulièrement sur les voûtes. Le passage de ce système décoratif sur les murs paraît plus original bien qu'il soit connu régionalement, à Seyssel par exemple . Le remplacement d'un décor à composition géométrique avec des motifs végétaux par un décor illusionniste (imitation de caissons) pourrait révéler un changement de mode dans les compositions picturales à la charnière des II $^{*}$ et III $^{*} \mathbf{s}$. Cette hypothèse reste à vérifier sur d'autres exemples datés précisément par les données archéologiques et le mobilier.

Le choix des motifs figurés dans le dernier état est assez traditionnel : d'une part, il est en rapport avec le monde de l'eau (monstres marins en relation avec

8. Rapport de fouilles de Seyssel, Service régional de l'Archéologie, 1980 . 


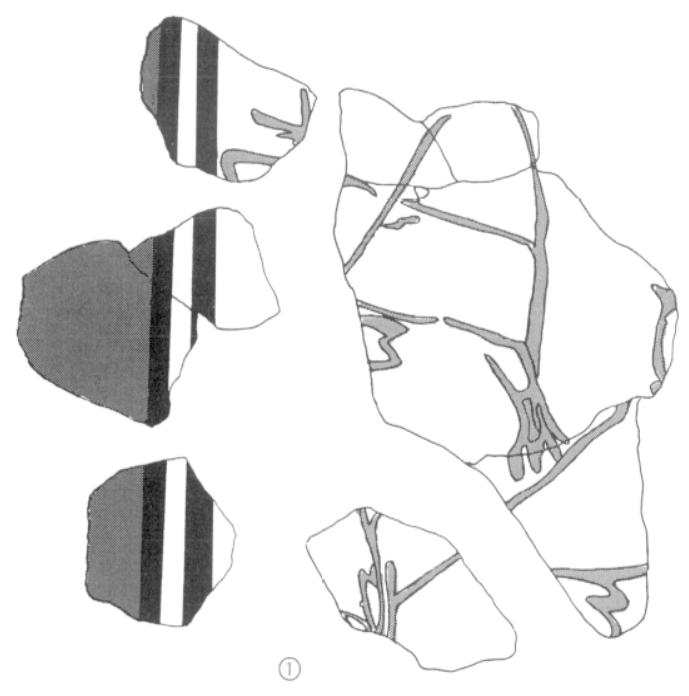

Fig. 13. 1 , décor VI-3, imitation de marbre; 2, décor VI-2, corniche d'entablement; 3, décor VI-2, encadrement de panneau et pilastre (dessin M.J. Bodolec).

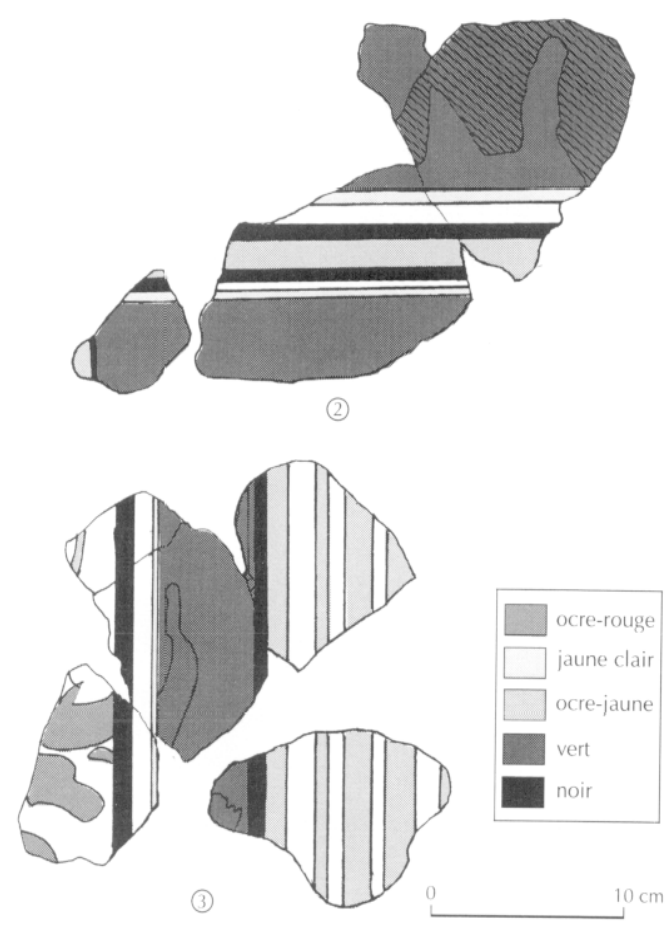

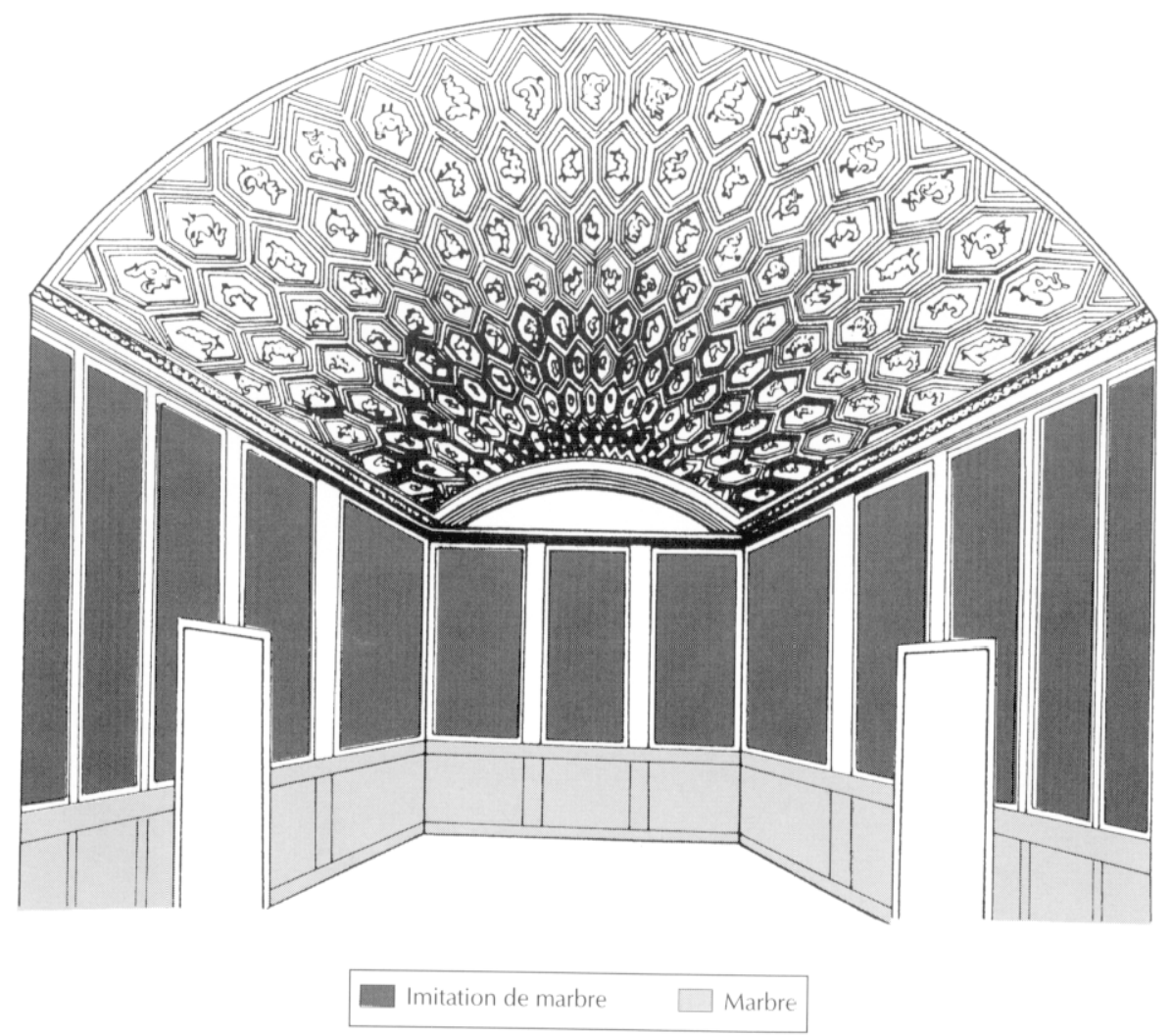

Fig. 14. Proposition de restitution du décor du tepidarium au délnut du III s. après.J.-C. (dessin O. Leblanc). 
Neptune, imitation d'opus musizum, incrustations et imitation de coquillages qui sont très fréquents dans la décoration des nymphées) ; d'autre part, la scène de lutte, à mettre en relation avec les jeux de la palestre (I.e Glay, 1983), est assez souvent représentéc sur des mosaïques de thermes et plus particulièrement sur une peinture de Saint-Romain-en-Gal qui ornait les latrines de thermes publics (Lcblanc, 1995). I.e choix des motifs figurés du décor de la première époque des thermes semble plus original, la symbolique étant plutôt d'inspiration dionysiaque. Toutefois un exemple très proche de masque a été reconnu dans un décor issu également de pièces thermales en Suisse (Fuchs, 1995) et nous connaissons des compositions dionysiaques en mosaïque dans le frigidarium des " thermes des Protomés " à Thuburbo-Maius (Poinsot, Quoniam, 1953) et en Grèce ${ }^{9}$.

L'absence de fragments d'enduits peints qui se rapporteraient à un décor de plinthe tend à confirmer l'existence de placages de marbre en partie basse de la paroi. Mais du fait de l'arasement des murs, aucun fragment n'en a été retrouvé. Cependant, l'abondance des fragments de placage en marbre dans les couches d'abandon du site corroborerait l'utilisation d'un tel matériau dans le décor pariétal aux II' et III's.

\footnotetext{
9. G. Touchais, Chronique... 1987, Macédoine, Thrace, Bulletin de Correspondance Hollénique, 112/2, 1988, p. 647. En 1987, lors de la fouille des thermes, est découverte, dans une salle de $100 \mathrm{~m}^{2}$, unc mosaïque polychrome de très grande qualité dont le panneau central porte un triomphe de Dionysos (époque des Sévères), Bulletin de l'Association internationale pour l'Étude de la Mosaïque antique, 113, 1990-1991, 1443, p. 218.
}

* 12, rue de la Comète, F-75007 Paris.

\section{ADDENDUM Note à propos de la flûte de Pan Annie BEIIS*}

L'identification de l'objet représenté ici n'offre aucune difficulté. Il s'agit presque à coup sûr d'une flûte de Pan, appelée syrinx par les auteurs latins, et dont l'origine grecque est bien attestée ne serait-ce que par le nom de l'instrument lui-même (fig. 5, n"2). Cette syrinx a ici la forme d'une aile d'oiseau : un petit côté horizontal, qui correspond à l'embouchure des tuyaux par laquelle s'effectuait l'insufflation; deux grands côtés verticaux, d'inégale longueur ; enfin, un quatrième côté, d'abord à peu près rectiligne, pour ensuite former une courbe qui rejoint le côté vertical le plus court. Sur de nombreuses représentations figurées, les flûtes de Pan sont constituées de sept ou huit tuyaux en roseau, parfaitement apparents, ligaturés par un lien ou une sorte de bandeau assez large (c'est alors le type même de la syrinx polycalame dont parlent les textes antiques). Ici, au contraire, l'artiste a grisé toute la surface de l'instrument (tout en suggérant peut-être par une teinte plus rougeâtre le "bandeau " de la flûte) sans même esquisser de façon sommaire l'alignement des tuyaux. Il y a toutes les chances qu'il l'ait fait parce qu'il avait en vue, non pas la flûte de Pan polycalame à tuyaux apparents, si fréquemment figurée entre les mains du dieu Pan ou des bergers, mais bien un instrument monoxyle, dont les tuyaux, de longucur décroissante, sont forés directement dans l'intérieur d'une pièce de bois et dont l'accord est affiné par un remplissage habilement dosé de cire. C'est ce qui explique l'arrondi de l'un des côtés. Un exemplaire de syrinx monoxyle a été découvert dans le puits d'une maison à Alésia en 1906 et se trouve aujourd'hui au Muséc des Antiquités Nationales de Saint-Germain-enLaye (Vendriès, 1993-1994) ; comme ici, le décor de sa face extérieure rappelle, par une série de traits horizontaux, l'ancien système de ligatures qui maintient ensemble les tuyaux. Généralement associée aux divinités champêtres, la syrinx pouvait également être un des accessoires des cérémonies dionysiaques. 


\section{BIBLIOGRAPHIE}

\section{BARBET A.}

1983a : La diffusion du 3c style pompéien en Gaule, Gallia, 41, p. 128-130.

1983b : Quelques rapports entre mosaique et peinture murale à l'époque romaine, in : Mosaïque, Recueil d'Hommages à Henri Stern, Paris, éd. Recherches sur la Civilisation, p. 50.

\section{DRACK W.}

1982 : Les peintures romaines en Suisse trouvées depuis 1950, in : La peinture murale romaine dans les provinces de L'Empire, Actes des Journées de Paris 1982, BAR International Series, 165, p. 16-23.

FUCHS M.

1995 : Voûte peinte à Vallon, in : Actes des
Séminaires de l'Association française de peintures murales antiques, Narbonne 1991, 10 $\mathrm{n}^{\circ}$ spécial à la Revue Archéologique de Picardie.

LEBL.ANC O.

1995 : Le décor des latrines des thermes des Lutteurs à Saint-Romain-en-Gal, in : Actes des Séminaires de l'Association française de peintures murales antiques, Chartres 1993, 10* n" spécial à la Revue Archéologique de Picardie.

LE GLAY M.

1983 : Hercule et la Iuuentus viennoise : à propos de la mosaïque des athlètes vainqueurs, in : Moscäque, Recueil d'Hommages à Henri Stern, Paris, éd. Recherches sur la Civilisation, p. 266, note 6.
Poinsot L., QuONIAM P.

1953 : Mosaïques des Bains des Protomés à Thuburbo-Majus, Karthago, p. 155167.

Savay-Guerraz H., Delaval E., Prisset J.-L., LeblanC O., BRISSAUd L.

1992 : Recherches archéologiques à SaintRomain-en-Gal (1988-1990), Bulletin de la Société des Amis de Vienne, 87, p. 17-25.

VENDRIÈs C.

1993-1994 : Le carnyx et la lyre, archéologie musicale en Gaule celtique et romaine, Catalogue d'exposition, $\mathrm{n}^{\circ} 118$, p. 88. 

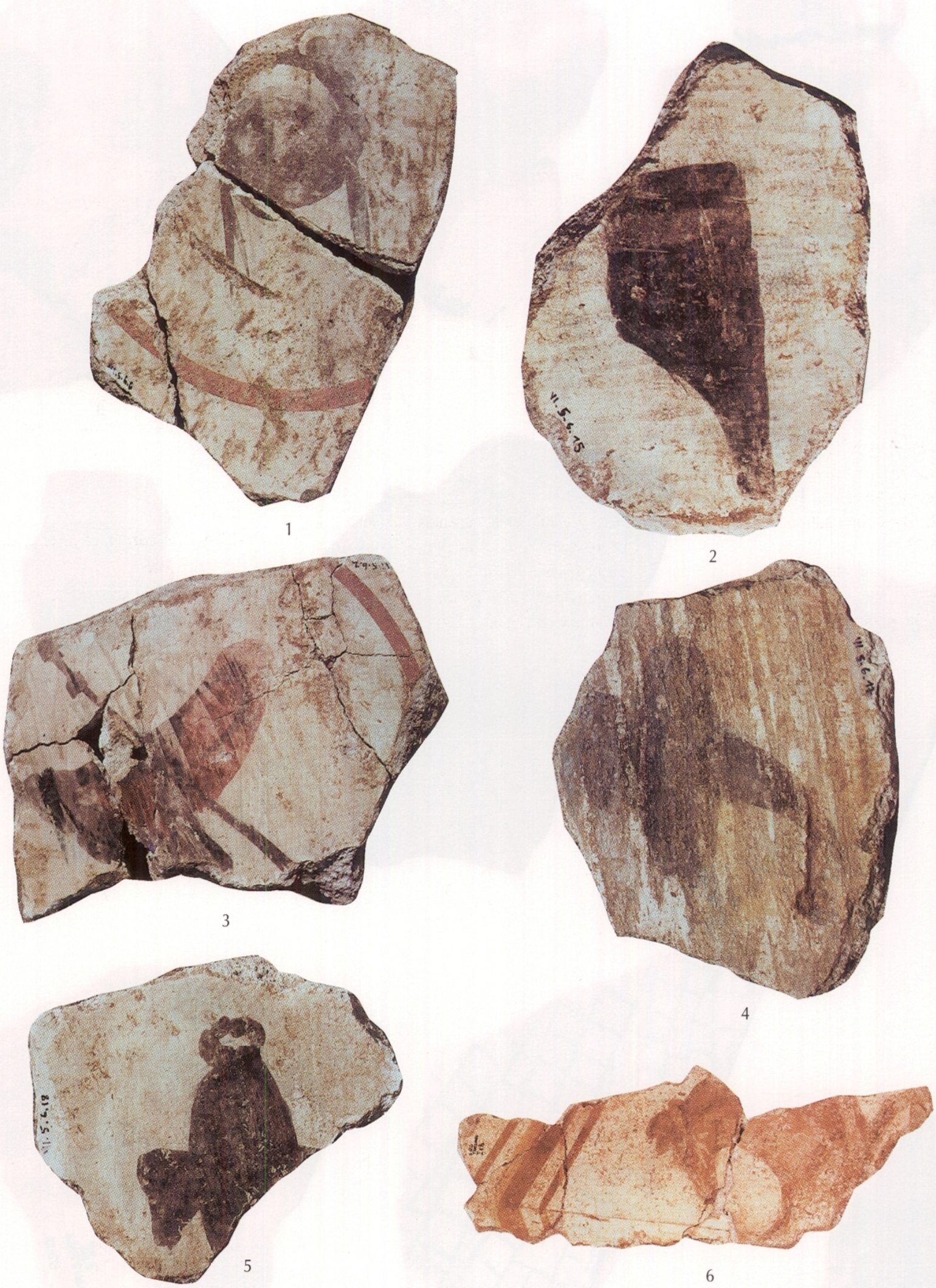

6

Pl. I. Décor VI-5 (nos 1 à 5); décor VI-1, décor aux hexagones ( $n^{\circ}$ 6). l, masque ; 2, flûte de Pan ; 3, phallus et thyrse ; 4, 5, gong ; 6, monstre marin. Sans échélle ( $n^{o s} 1-5$, photos P. Veyssere et O. Leblanc, ; $n^{0} 6$, photo M.J. Bodolec et A. Lebot). 